\section{Asthmaschulung für Lehrer - Notwendigkeit und Möglichkeiten}

Zusammenfassung: Lehrer und Schulen sind zunehmend mit der Betreuung von Kindern/Jugendlichen mit Asthma bronchiale konfrontiert, ohne darauf in ihrer Ausbildung vorbereitet worden zu sein: Jeder Lehrer hat pro Schulstunde ca. 2 bis 4 asthmabetroffene Kinder/Jugendliche zu betreuen. Die Lehrer/ Schulen fühlen sich meist nicht ausreichend informiert bezüglich eines adäquaten Umgangs mit dem Asthma bronchiale. Methodik: 1999/2000 wurde in Osnabrück eine Befragung hinsichtlich des möglichen Defizits an Schulen durchgeführt, in Anlehnung an eine Voruntersuchung aus einer Wiener Arbeitsgruppe. Die Untersuchung zeigte im Wesentlichen vergleichbare Defizite in Bezug auf Kenntnisse und Einschätzung der Asthmasituation auf. Parallel zu dieser Befragung erfolgte an 40 anderen Schulen/Kindergärten und Seminaren $(n=648$ Teilnehmer) eine Intervention über ein 3-stündiges Seminar mit den Themen „Was ist Asthma?“, „Selbsteinschätzung“, „Auslösevermeidung“, „Asthma und Sport“, „Notfallmanagement“. Die Seminare wurden abgehalten direkt in den Schulen, möglichst komplette Kollegien sollten daran teilnehmen. Die Resonanz auf das Angebot war so groß, dass nicht alle Teilnahmewünsche erfüllt werden konnten. Fazit: Die vermittelten Inhalte und auch praktischen Techniken waren gut lernbar und aus Sicht der Lehrer für eine Umsetzung im Schulalltag geeignet, die Teilnehmer fühlten sich nach dem Seminar kompetenter im Umgang mit dem Asthma bronchiale. Derartige Seminare scheinen geeignet, Schulen/Lehrer/Erzieher mit einem vertretbaren Aufwand fortzubilden. Es ist zu überprüfen, ob - zumindest für die häufigsten chronischen Erkrankungen - derartige Fortbildungsangebote bereits in das Referendariat integriert werden sollten.

Dealing with Asthma for Teachers - Need and Possibilities: Teachers and schools are confronted with an increasing number of children with asthma. Teachers have to care for 2 to 4 children or adolescents with asthma in an average class; but they feel that they are not adequately informed to cope with such problems. Methods: In 1999 and 2000, we conducted an inquiry in schools in the region of Osnabrueck. This investigation used approximately the same tools as a previous inquiry in Vienna. Results were similar and demonstrated deficits in the knowledge about asthma and in the ability to manage children with asthma exacerbations. At the same time, we offered a three-hour-programme in $\mathbf{4 0}$ schools and nurseries, to be given

Pneumologie 2001; 55: $512-519$

(C) Georg Thieme Verlag Stuttgart · New York ISSN 0934-8387
R. Szczepanski, G. Brockmann, G. Friede

Kinderhospital Osnabrück (Leiter: Prof. Dr. K. E. v. Mühlendahl) in the rooms of the schools. We always tried to reach all teachers of one school. Topics were: What is asthma? Self grading of severity; asthma and sports; emergency management; avoidance of triggers. Results: There were 648 participants. There was much interest in the seminars, and we could not always admit all applicans. Theoretical and practical contents taught were, as judged by the participants, helpful in the average school settings. The participants felt that they were now better prepared and more competent in dealing with bronchial asthma in their pupils. Conclusion: We conclude that such seminars are helpful for teachers. The program can be conducted with moderate financial and man power resources. One may aim at including such educational units into the regular curriculum of teachers, at least as far as the most common chronic childhood and adolescent diseases are concerned.

\section{Einleitung}

Asthma bronchiale stellt mit einer Prävalenz von etwa $10 \%$ $[1,2]$ die häufigste chronische Erkrankung im Kindes- und Jugendalter dar. Die Weiterentwicklung der Medikamente und Optimierung der therapeutischen Stufenpläne einerseits sowie eine strukturierte, qualifizierte Schulung von Kindern/ Jugendlichen und deren Eltern andererseits sind Bausteine, die heute ein adäquates Management von Akut- und Dauertherapie im ambulanten Bereich ermöglichen [3-7]. In geschulten Familien ist der Anteil der Fehltage in der Schule seltener, so dass zunächst einmal unterstellt werden kann, dass die Verbesserung der medizinischen Versorgung und auch die Asthmaschulungsmaßnahmen unmittelbar zu einer Besserung der schulischen Situation der Kinder und Jugendlichen geführt haben [8-10].

Einen Großteil des Tages verbringen die Kinder und Jugendlichen in der Schule (Unterricht, Ausflüge, Klassenfahrten), somit fehlt während vieler Stunden die Unterstützung der Eltern im Umgang mit der Dauertherapie und Akutinterventionen. Auch bei Jugendlichen gilt dies gleichermaßen, da Gleichaltrige in aller Regel auch nicht adäquat reagieren können [11]. Bei einer angenommenen Klassengröße von 30 Kindern hat jeder Lehrer in jeder Unterrichtsstunde zwei bis vier Kinder mit Asthma bronchiale zu betreuen, eine Situation, auf die Lehrer in der Regel nur dann vorbereitet sind, wenn sie entweder aus dem eigenen Familien-/Bekanntenkreis über Kenntnisse mit Asthma bronchiale verfügen oder aber sich aus anderen Gründen gezielt weitergebildet haben. 
$70 \%$ der Kinder haben Belastungsasthma, was insbesondere für den Sportunterricht in der Schule und auch für die Pausen relevant ist [12]. Lecheler und Gauer [13] erfassten die Schuldefizite durch Asthma bronchiale bei 186 Kindern: Nur 27,4\% hatten keine nennenswerten Schulausfallzeiten; 21,5\% hatten bis zu $10 \%, 12,9 \%$ hatten $10-20 \%, 12,9 \%$ hatten 20 $30 \%$ und $17,7 \%$ hatten eine Ausfallzeit von über $30 \%$ der Schultage. Der Schulfortschritt war nur bei 19,4\% nicht beeinträchtigt; 30,8\% hatten ein Jahr, 26,3\% hatten zwei Jahre, 8,1\% hatten drei Jahre und 1,6\% hatten vier Jahre verloren. 25,3\% der Schüler nahmen nie am Schulsport teil. Im Rahmen der Multizenteruntersuchung über die Wirksamkeit der Asthmaschulung bei Kindern und Jugendlichen $[8,9]$ gaben die Familien an, dass die Kinder 7 bis 9 Tage pro Jahr in der Schule gefehlt hatten.

Das Wissen über Asthma bronchiale war bei der Untersuchung von Bevis und Taylor [14] ungenügend. Nur ein Drittel der Lehrer kannte die Möglichkeit einer Prämedikation vor Sport, $21 \%$ waren sich nicht sicher, ob eine Teilnahme am Sport möglich sei oder hielten sie für unmöglich. 39\% wollten das Mitführen eines Inhalators verbieten, 95\% gaben selbst an, unzureichend informiert zu sein. In einer Untersuchung an 216 Schulen in Südwales [15] wurden die Schulleiter befragt, 191 (=88\%) antworteten: 17\% der Schüler hatten im Schnitt 9 Tage (2-16) wegen Asthma gefehlt. Nur 76 (=40\%) der Schulen gestatteten Kindern ihren Inhalator zu benutzen; $12(=6 \%)$ forderten Eltern auf, den Inhalator zu bedienen; in 115 (=60\%) der Schulen glaubten die Leiter, dass das Kollegium mit dem Asthmamanagement vertraut ist. Dem gegenüber hielten 174 (= 91\%) der Schulleiter ein Krankheitstraining für wünschenswert. 108 der Schulen $(=62 \%)$ hatten keine Anweisungen/schriftliche Instruktionen für die Asthmatherapie; $168(=88 \%)$ hatten keine Information über weiter behandelnde Ärzte und nur 31 (=16\%) hatten Notfallmedikamente für den Asthmaanfall vorrätig. French et al. [16] kamen zu ähnlich ungünstigen Daten bei einer Erhebung an 164 Grundschulen in Westaustralien. Gibson et al. [11] beschrieben das gleiche Dilemma für Lehrer und auch für die Klassenkameraden bei Jugendlichen.

Stohlhofer et al. [17] befragten 1311 Wiener Volksschullehrer bezüglich ihres Wissensstandes (1054 Antworten). Nur 1\% der befragten Lehrkräfte gab an, asthmabetroffene Kinder in der Klasse zu haben; $10 \%$ der befragten Lehrer waren bereits im Rahmen des Unterrichtes mit einem akuten Asthmaanfall konfrontiert. Nur 2\% der Lehrer hatten bereits einmal ein Asthmaschulungsprogramm durchlaufen. Lehrer, die ein Trainingsprogramm durchlaufen hatten, ließen Kinder signifikant häufiger am Schulsport teilnehmen. 80\% der Lehrer waren bereit, asthmabetroffenen Kindern bei der Medikamenteneinnahme zu helfen, aber nur 30\% gaben an, im Umgang mit inhalativen Medikamenten vertraut zu sein. Das allgemeine Basiswissen über asthmaspezifische Symptome war gut, aber gering bezüglich Therapie und Auslösern. Nur 45\% kannten den Effekt für eine präventive Medikation.

Einige Autoren [15-17] fordern als Fazit ihrer Untersuchung die Durchführung von Asthmaschulung für Lehrer. Ziel unserer Untersuchung war 1. den Wissensstand bei Lehrern verschiedener allgemein bildender Schulen im Raum Osnabrück und Umgebung zu erfragen sowie 2. an allgemein bildenden Schulen, die nicht an dieser Wissensbefragung teilnahmen, eine Intervention im Sinne einer Asthmaschulung für Lehrer durchzuführen, mit dem Ziel, den Informationsstand der Lehrer und Interventionsmöglichkeiten an Schulen und Kindergärten dadurch zu verbessern.

\section{Methodik}

1. Erhebung des Wissensstandes: Mit Genehmigung der Autoren [17] wurden im Januar 2000 über 1000 Fragebogen von der Bezirksregierung Weser-Ems an allgemein bildende Schulen versandt. In den ersten 3 Monaten nach der Aussendung wurden 483 auswertbare Bogen zurückgeschickt. Schulen, die an dieser Befragung teilnahmen, erhielten keine Intervention.

2. Im Rahmen eines Pilotprojektes $[18,19]$ wurde die prinzipielle Durchführbarkeit einer Schulungsmaßnahme bezüglich Asthma bronchiale für Lehrerkollegien im Hinblick auf Zeitumfang, Inhalte, Akzeptanz an 8 Schulen erprobt. Neu war dabei, dass die Kollegien die Fortbildung in den Schulen selbst angeboten bekamen. Der Stundenplan und die Durchführungsmodalitäten wurden aufgrund der Teilnehmerrückmeldungen weiterentwickelt.

Das Seminar umfasst 3 Zeitstunden (s. Tab.1), insbesondere einen Notfallplan (s. Abb.1 u. 2). Die Themen wurden von Asthmatrainern der Akademie „Luftiku(r)s“ Osnabrück durchgeführt. Inhalte und methodisch-didaktische Umsetzung entsprachen dem Elternseminar der Patientenschulung bei Asthma bronchiale im Kindes- und Jugendalter [7,20,21]. Die Weiterbildung erfolgt im interaktiven Gespräch, ergänzt durch möglichst viele praktische Übungen. Die Rekrutierung der teilnehmenden Kollegien erfolgte nach Bekanntgabe der Projektes durch die Bezirksregierung Weser-Ems bzw. über die direkte Anfrage von den Schulen/Kindergärten bei der Akademie „Luftiku(r)s“. Die Fortbildung wurde für ganze Kollegien angeboten; je 15 bis 20 Lehrer war ein Trainer zugegen, so dass immer eine Kleingruppenarbeit gewährleistet werden konnte. Aus organisatorischen Gründen war eine Versendung des Fragebogens [nach 17] vor dem Seminar nicht möglich. Die Befragung der Teilnehmer unmittelbar am Ende des Seminars erfolgte über einen eigens entwickelten Fragebogen $[18,19]$.

Tab. 1 Stundenplan des Lehrerseminars

$\begin{array}{ll}15 \mathrm{~min} & \text { Begrüßung, Erfahrungen mit Asthma in der Schule } \\ 45 \mathrm{~min} & \text { "Was ist Asthma?“, Strohhalmübung } \\ 15 \mathrm{~min} & \text { Selbsteinschätzung (Lungendetektiv, Peak Flow) } \\ 30 \mathrm{~min} & \text { Pause, evtl. Rundgang durch die Schule } \\ 45 \mathrm{~min} & \text { Auslöser/Auslösevermeidung } \\ 30 \mathrm{~min} & \begin{array}{l}\text { Notfallmanagement, atemerleichternde Stellungen, } \\ \text { Spraygebrauch }\end{array}\end{array}$

Die Auswertung der Defizitanalyse erfolgte nach den gleichen Kriterien wie bei Stohlhofer [17]. Die Darstellung der Ergebnisse erfolgt in Prozent, so dass die Studien miteinander verglichen werden können. 


\section{SCHÜLER \\ ASTHMA - NOTFA LLPLAN}
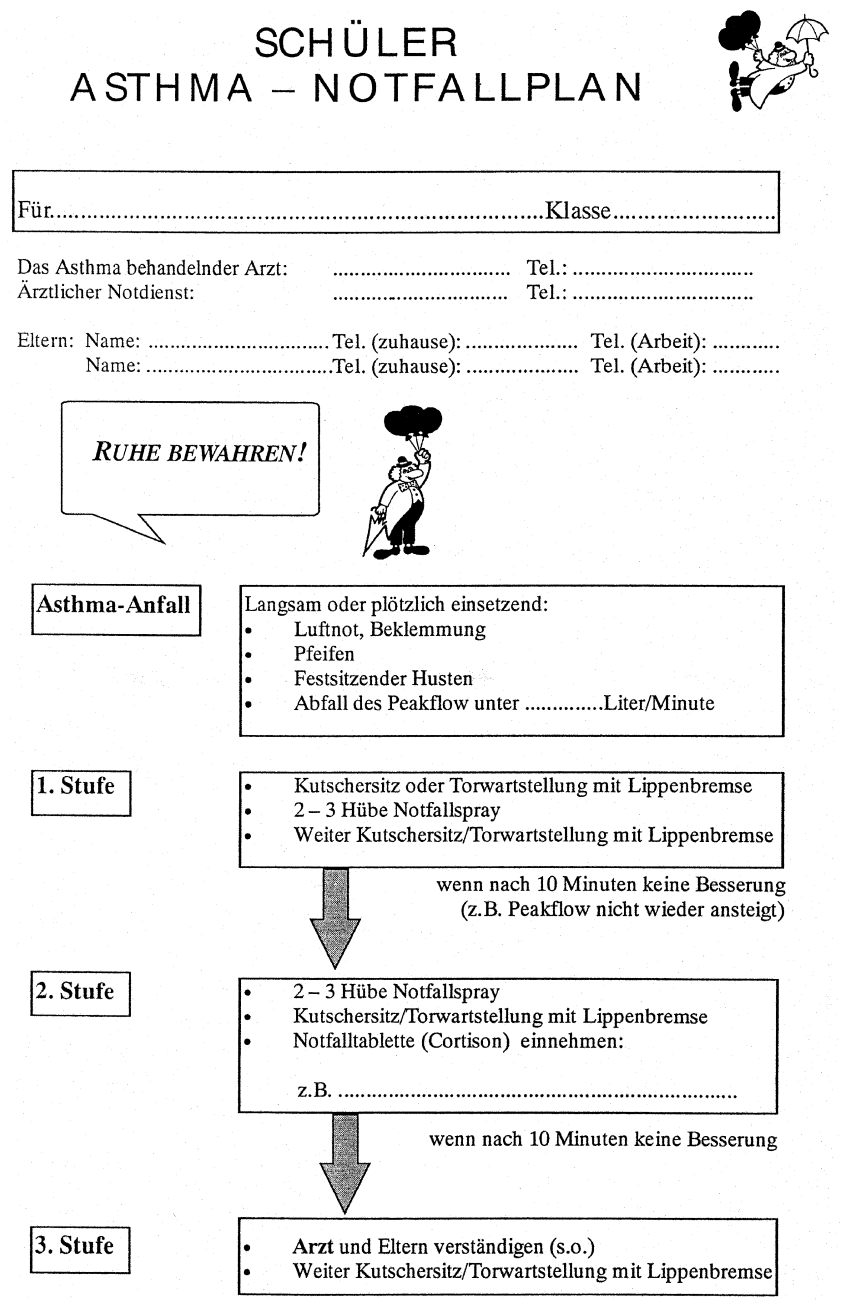

Abb. 1 Schüler-Asthma-Notfallplan.

\section{Ergebnisse}

\section{A - Defiziterfassung}

Die Antworten wurden ausgewertet aufgrund von 483 auswertbarer Fragebogen (97 Lehrer $=20 \%$ von Grundschulen, $262(=54 \%)$ von Orientierungsstufen/Sekundarstufen I; 76 (=16\%) von Gymnasien; 13 (=3\%) Gesamtschulen; 27 (=5\%) Sonderschulen; 8 (=2\%) Berufschulen.

Die Daten hinsichtlich der Defizite (Themenbereiche, Allgemeinwissen über Asthma bronchiale, Symptome und Auslöser, Asthma und Sport, Therapiemöglichkeiten, eigene Erfahrungen mit Asthma bronchiale) entsprachen denen der Wiener Arbeitsgruppe, obwohl sie an Schulen unterschiedlicher Art sowie an einer insgesamt kleineren Gruppe erhoben wurden. In den Tab. 2-6 sind die Prozentzahlen der Befragung in Osnabrück angegeben; die entsprechenden Prozentzahlen der Wiener Untersuchung [17] sind jeweils in Klammer gesetzt.

In einer separaten Auswertung (tabellarisch nicht dargestellt) wurden die Antworten derjenigen Lehrer (64/483 = 13\%) untersucht, die sich selbst in der Befragung als ausreichend

Bitte von den Eltern und/oder dem behandelnden Arzt ausfüllen lassen

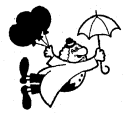

Auslöser:

$\square$ Körperliche Belastung

$\square$ Zigarettenrauch

$\square$ Infekte

ㅁ Tiere:...

Pollen:

P Haustabmilbe:

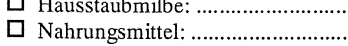

Tägliche Dauermedikamente:

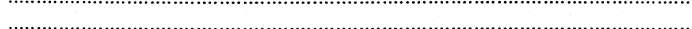

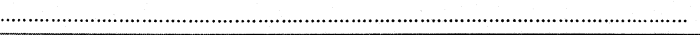

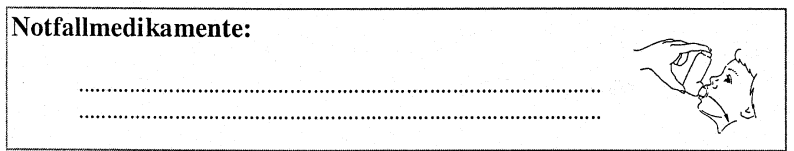

Spezielle Maßnahmen in der Schule zur Verhütung von Asthmaverschlechterungen (insbesondere bzgl. Sport):

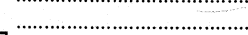

$\square$ Medikamente vor Sport:

rechten Gebrauch seiner Medikamente geschult. Das Kind sollte seine Notfallmedikamente mit sich führen und selbständig benutzen dürfen.

Unterschrift der Eltern

Datum

Unterschrift des behandelnden Arztes

Datum

Abb. 2 Rückseite des Schüler-Asthma-Notfallplanes.

informiert darstellten („Glauben Sie ausreichend über Asthma bronchiale informiert zu sein?“). In dieser Subgruppe ergaben sich keine signifikanten Unterschiede im Antwortverhalten im Vergleich zum gesamten Kollektiv.

\section{$B$ - Intervention/Lehrerseminar}

Die finanziellen Mittel erlaubten die Durchführung von 40 Seminaren (bei mehr als 20 Lehrern in einem Kollegium zwei Parallelseminare durch zwei Trainer). Die Schulen hatten meist von dem Angebot über Schulleiter-Dienstbesprechungen erfahren sowie über Informationsschreiben der Bezirksregierung. Der Entschluss zur Teilnahme am Seminar erfolgte zudem aufgrund von Hinweisen von Eltern aus Asthmaschulungskreisen sowie Informationen von Lehrern, die bereits an anderen Schulen an diesem Seminar teilgenommen hatten (s. Tab. 7).

Im Verlauf des Schuljahres 1999/2000 wurden an 40 Institutionen (Schulen, Kindergärten, Ausbildungsseminare) die Fortbildungsseminare durchgeführt. Dabei wurden 648 Lehrer und Erzieher erreicht. 90,6\% der teilnehmenden Personen füllten den Fragebogen bezüglich der Umsetzbarkeit des 
Tab. 2 Eigene Erfahrungen mit Asthma bronchiale - Osnabrücker Erhebung n = 483, Angaben in \%. In Klammern Wiener Studie n = 1054, Angaben in \%

\begin{tabular}{|c|c|c|c|}
\hline & ja & nein & weiß nicht \\
\hline Glauben Sie ausreichend über Asthma bronchiale informiert zu sein? & $13(6,0)$ & $83(94,0)$ & 4 \\
\hline Haben Sie im Rahmen eines Erste-Hilfe-Kurses die Asthmaakutversorgung erlernt? & $5(5,3)$ & $92(94,7)$ & 3 \\
\hline Haben Sie jemals an einer Asthmaschulung teilgenommen? & $4(2,2)$ & $93(97,8)$ & 3 \\
\hline $\begin{array}{l}\text { Würden Sie asthmatische Kinder bei der Einnahme ihrer Medikamente oder Benutzung ihres } \\
\text { Asthmasprays beaufsichtigen? }\end{array}$ & $78(81,7)$ & $16(18,3)$ & 6 \\
\hline $\begin{array}{l}\text { Würden Sie im Akutfall bis zum Eintreffen des Notarztes dem Kind ein Asthmaspray } \\
\text { verabreichen? }\end{array}$ & $78(69,4)$ & $14(30,6)$ & 8 \\
\hline Gibt es in Ihrer Klasse Kinder mit bekanntem Asthma bronchiale? & $36(1,0)$ & $59(99,0)$ & 5 \\
\hline Trat jemals im Unterricht bei einem Kind ein Asthmaanfall auf? & $23(9,4)$ & $74(90,6)$ & 3 \\
\hline Trat jemals im Rahmen des Sportunterrichtes bei einem Kind ein Asthmaanfall auf? & $24(5,1)$ & $61(94,9)$ & 15 \\
\hline Sind Sie selbst Elternteil eines asthmakranken Kindes? & $6(3,4)$ & $92(96,6)$ & 2 \\
\hline Gibt es Asthma bronchiale in Ihrer Verwandtschaft? & $30(17,7)$ & $67(82,3)$ & 3 \\
\hline Leiden Sie selbst an Asthma bronchiale? & $8(3,3)$ & $90(96,7)$ & 2 \\
\hline
\end{tabular}

Tab. 3 Allgemeinwissen über Asthma bronchiale - Osnabrücker Erhebung n = 483, Angaben in \%. In Klammern Wiener Studie n= 1054, Angaben in $\%$; richtige Antworten fett gedruckt

\begin{tabular}{|c|c|c|c|c|}
\hline & richtig & falsch & weiß nicht & $\begin{array}{l}\text { keine } \\
\text { Antwort }\end{array}$ \\
\hline Ungefähr $10 \%$ der deutschen Kinder leiden an Asthma bronchiale & $61(45,3)$ & $8(11,1)$ & $29(43,6)$ & 2 \\
\hline Asthmakranke Kinder haben überempfindliche Bronchien & $78(75,4)$ & $7(6,8)$ & $13(17,8)$ & 2 \\
\hline Unbehandeltes Asthma kann zu einer Wachstumsverzögerung führen & $34(28,4)$ & $25(21,6)$ & $39(50,0)$ & 2 \\
\hline Asthma ist infektiös & $4(2,3)$ & $87(87,8)$ & $7(9,9)$ & 2 \\
\hline Asthma schädigt das Herz & $56(60,4)$ & $16(10,7)$ & $27(28,8)$ & 1 \\
\hline Asthmakranke Kinder dürfen keine Milchprodukte essen & $4(4,6)$ & $69(64,9)$ & $24(30,5)$ & 3 \\
\hline Kinder können am Asthma sterben & $75(49,6)$ & $8(14,1)$ & $15(36,3)$ & 2 \\
\hline Asthma wird durch emotinale Faktoren beeinflusst & $89(91,1)$ & $3(2,1)$ & $7(6,8)$ & 1 \\
\hline Im Asthmafall kommt es zu einer akuten Verengung der Bronchien & $90(83,9)$ & $1(0,9)$ & $8(15,2)$ & 1 \\
\hline Asthmakranke Kinder kommen meist aus sozial mindergestellten Familien & $1(2,8)$ & $92(88,0)$ & $6(9,2)$ & 1 \\
\hline
\end{tabular}

Seminars im Schulalltag aus (Verteilung der Teilnehmer s. Tab. 8). Die Teilnehmer wurden auch nach ihren Vorerfahrungen mit Asthma bronchiale gefragt (s. Tab.9). Diese Fragen stammen aus dem Fragebogen nach Stohlhofer [17]; die Antworten entsprechen denen der Defizitanalyse.

Die Inhalte des Seminars (s. Abb.1) wurden hinsichtlich ihrer Umsetzbarkeit erfragt (s. Tab. 10 „Inwieweit glauben Sie die Informationen und Anregungen aus der Fortbildung in Ihrem Alltag umsetzen zu können?"). Der eigens für die Belange der Schulen entwickelte Notfallplan (s. Abb.1 u. 2) wurde hinsichtlich seiner Praktikabilität erfragt (,Stellen Sie sich eine Notfallsituation in Ihrer Schule vor. Welche folgenden [Organisation-]Schritte trauen Sie sich zu?“, s. Tab.11). Zudem wurden die Teilnehmer befragt, ob die Maßnahmen zur Betreuung asthmakranker Kinder im Schulalltag integrierbar sein können („Maßnahmen zur Betreuung asthmakranker Kinder müssen in den Schulalltag integrierbar sein. Wie sieht es mit der Umsetzung der folgenden Punkte an Ihrer Schule aus?“). Die Antworten sind der Tab.12 zu entnehmen.

\section{Diskussion}

Aus der englischsprachigen Literatur ist ein hohes Maß an Wissensdefiziten unter Lehrern bzw. Schulen berichtet worden [14-16]. Aus dem deutschsprachigen Raum liegt bisher nur die Untersuchung bei Wiener Vorschullehrern vor [17]. Die im ersten Teil unserer Untersuchung erfassten Daten weisen darauf hin, dass ein der Wiener Untersuchung vergleichbares Defizit im Raum Osnabrück bei Lehrern vorliegt, wobei im Raum Osnabrück alle allgemein bildenden Schulen angesprochen wurden.

Die Daten zeigen - mit der Einschränkung, dass bei der Untersuchung im Osnabrücker Raum das Kollektiv deutlich kleiner ist als in Wien (483 versus 1054) und von der Zusammensetzung nicht vergleichbar -, dass insgesamt von einer vergleichbar ungünstigen Ausgangslage hinsichtlich des Wissens bzw. der praktischen Fähigkeiten bezogen auf das Asthmamanagement durch Lehrer ausgegangen werden muss. Die zitierten und die eigenen Untersuchungen $\mathrm{zu}$ den Defiziten unterstreichen den Eindruck aus Asthmaschulungs- 
Tab. 4 Symptome und Auslöser von Asthma bronchiale - Osnabrücker Erhebung n=483, Angaben in \%. In Klammern Wiener Studie $n=1054$, Angaben in \%; richtige Antworten fett gedruckt

\begin{tabular}{|c|c|c|c|c|}
\hline & richtig & falsch & weiß nicht & $\begin{array}{l}\text { keine } \\
\text { Antwort }\end{array}$ \\
\hline \multicolumn{5}{|l|}{ Welche Symptome setzen Sie mit Asthma bronchiale in Verbindung? } \\
\hline nächtlicher Husten & $45(58,0)$ & $31(20,0)$ & $19(22,0)$ & 5 \\
\hline Husten am Ende einer körperlichen Belastung & $77(81,5)$ & $10(8,7)$ & $10(9,8)$ & 3 \\
\hline Husten nach dem Essen & $5(8,5)$ & $67(63,8)$ & $24(27,7)$ & 4 \\
\hline anfallartig auftretende Atemnot & $94(96,3)$ & $3(1,4)$ & $2(2,3)$ & 1 \\
\hline Fieber, Husten, Schwierigkeiten b. Einatmen & $28(40,9)$ & $48(34,9)$ & $20(24,2)$ & 4 \\
\hline stechende Schmerzen im Brustkorb mit Ausstrahlung in den linken Arm & $6(13,4)$ & $68(49,2)$ & $23(37,4)$ & 3 \\
\hline pfeifende, giemende Atemgeräusche & $89(84,6)$ & $3(4,3)$ & $5(11,1)$ & 3 \\
\hline Bei einem akuten Asthmaanfall ist die Einatmung erschwert & $68(75,5)$ & $22(11,3)$ & $6(13,2)$ & 4 \\
\hline Ausatmung erschwert & $47(33,2)$ & $29(37,4)$ & $16(29,4)$ & 8 \\
\hline \multicolumn{5}{|l|}{$\begin{array}{l}\text { Welche Situationen/Umstände können einen Asthmaanfall auslösen oder ein } \\
\text { bestehendes Asthma verschlimmern? }\end{array}$} \\
\hline Erkältungskrankheit & $82(76,2)$ & $4(7,0)$ & $11(16,8)$ & 3 \\
\hline kalte Luft & $31(34,2)$ & $26(25,9)$ & $29(39,9)$ & 4 \\
\hline warme Luft & $31(16,5)$ & $28(35,7)$ & $37(47,8)$ & 4 \\
\hline körperliche Anstrengung & $88(88,4)$ & $4(2,5)$ & $6(9,1)$ & 2 \\
\hline heißes Bad & $13(12,0)$ & $42(40,0)$ & $41(48,0)$ & 4 \\
\hline Aufenthalt in verrauchten Räumen & $93(89,4)$ & $1(2,1)$ & $5(8,5)$ & 1 \\
\hline blühende Wiesen und Felder & $66(73,6)$ & $17(9,3)$ & $16(17,1)$ & 1 \\
\hline direkter Kontakt zu Tieren & $67(71,4)$ & $16(9,9)$ & $15(18,7)$ & 2 \\
\hline Spielen im Regen & $5(5,0)$ & $68(64,3)$ & $24(30,7)$ & 3 \\
\hline
\end{tabular}

Tab. 5 Asthma und Sport - Osnabrücker Erhebung n = 483, Angaben in \%. In Klammern Wiener Studie n = 1054, Angaben in \%; richtige Antworten fett gedruckt

richtig falsch weiß nicht keine

Glauben Sie, dass asthmakranke Kinder im Unterricht

genauso belastbar wie gesunde Kinder sind

nicht so intelligent wie gesunde Kinder sind

schwieriger als gesunde Kinder sind

Asthmakranke Kinder können nicht bei sportlichen Wettkämpfen mitmachen

Die volle Teilnahme asthmakranker Kinder am Sportunterricht ist anzustreben

Durch die Einnahme entsprechender Medikamente vor sportlicher Betätigung kann ein Anfall verhindert werden

Asthmakranke Kinder dürfen schwimmen

Asthmakranke Kinder können an Wandertagen, Klassenfahrten teilnehmen

Mit der richtigen Behandlung kann ein asthmakrankes Kind ein Leben ohne Einschränkungen führen

$\begin{array}{llll}\mathbf{4 5}(\mathbf{4 7 , 0 )} & 42(38,2) & 11(14,8) & 2 \\ 2(3,6) & \mathbf{9 2 ( 9 4 , 8 )} & 1(1,6) & 5 \\ 9(15,3) & \mathbf{7 8 ( 7 2 , 1 )} & 7(12,6) & 6 \\ 10(23,4) & \mathbf{7 6 ( 5 0 , 6 )} & 12(26,0) & 2 \\ \mathbf{7 6 ( 5 7 , 1 )} & 12(19,3) & 11(23,6) & 1 \\ \mathbf{5 4}(\mathbf{4 4 , 6 )} & 13(7,6) & 31(47,8) & 2 \\ \mathbf{9 1 ( 8 0 , 5 )} & 2(0,9) & 6(18,6) & 1 \\ \mathbf{9 8}(\mathbf{9 2 , 3 )} & 0(1,9) & 1(5,8) & 1 \\ \mathbf{7 8 ( 7 7 , 7 )} & 10(5,0) & 11(17,3) & 1\end{array}$


Tab. 6 Therapiemöglichkeiten des Asthma bronchiale - Osnabrücker Erhebung n=483, Angaben in \%. In Klammern Wiener Studie $n=1054$, Angaben in \%; richtige Antworten fett gedruckt

richtig falsch weiß nicht keine

Welche Körperposition würden Sie als atemerleichternd bezeichnen?

sitzend mit aufgestützten Armen

auf dem Rücken liegend

$68(66,1) \quad 11(12,4) \quad 15(21,5) \quad 6$

in Seitenlage liegend

$10(10,9)$

$25(38,7)$

Inhalative Medikamente haben weniger Nebenwirkungen als die Einnahme in Tablettenform

Antibiotika sind in der Asthmabehandlung unentbehrlich

Kortison ist in der Basisbehandlung ein sehr wertvolles Medikament

Kennen Sie ein Asthmamedikament namentlich?

$45(44,9)$

$8(5,4)$

$44(28,4)$

$24(17,6)$

$37(31,7)$

Sind Sie im Umgang mit einem inhalativen Medikament vertraut?

$18(11,2)$

$11(12,4)$

$60(69,0)$

$18(20,1)$

$34(28,2)$

$30(33,1)$

12

$14(6,1)$

$35(49,0)$

$48(38,0)$

$39(56,6)$

$34(53,6)$

$16(18,0)$

$72(82,4)$

$59(68,3)$

$78(88,8)$

keine

Antwort

Wissen Sie, was ein Peak-Flow-Meter ist?

- Häufig trauen die Kinder sich nicht, die Dauertherapie oder auch eine notwendige Akutintervention einzusetzen bzw. es wird seitens des betreffenden Lehrers nicht gestattet.

- Im Sportunterricht sind angemessene Belastungspausen oft nicht möglich („Simulant“).

- Die Lehrer sind häufig überfordert, beginnende Asthmasymptome zu registrieren und dem Kind den Freiraum für eine mögliche Selbsthilfe zu ermöglichen.

- Gelegentlich werden Kinder ohne eine primäre medikamentöse Versorgung direkt nach Hause geschickt, teils ohne Begleitung. Daneben gibt es häufig Kommunikationsprobleme zwischen Lehrer und Eltern über Krankheit und Therapie, aber auch Kommunikationsprobleme bezüglich des Umganges mit Asthma bronchiale innerhalb von Lehrerkollegien.

Über viele Jahre ist von unserer Arbeitsgruppe versucht worden, über Vorträge für Sportobleute bzw. Schulleiter die Situation in den Schulen zu verbessern, ohne dass ein ausreichender Effekt registriert werden konnte. Die Teilnahmefrequenzen an derartigen Fortbildungen war meist gering aus den verschiedensten organisatorischen oder persönlichen Gründen der einzelnen Lehrer. Darüber hinaus ist es sicherlich für Lehrer schwierig, medizinische Inhalte eines Vortrages innerhalb des jeweiligen Kollegiums angemessen $\mathrm{zu}$ implementieren. Aus diesem Grunde wurde zunächst über ein Pilotprojekt 1996/1997 versucht, ob eine „Bringestruktur“ eine bessere Askzeptanz ermöglicht. Von 9 angesprochenen Schulen innerhalb einer nordrhein-westfälischen Kleinstadt hatten 8 spontan an einem Asthmafortbildungsseminar für Lehrer teilgenommen und zwar mit den gesamten Kollegien. Nach Überprüfung der Praktikabilität des Seminarangebotes (Teilnahme möglichst des gesamten Kollegiums, Kleingruppenarbeit, praktisches Üben, angemessene Inhalte, handlungsrelevantes Wissen) erfolgte dann die Überprüfung der Effekte des Seminars an 40 Einrichtungen (allgemein bildende Schulen, Seminare und Kindergärten). Die Intervention erfolgte im Zeitraum des Schuljahres 1999/2000. Die Nachfrage nach einer Teilnahme durch Schulen, Kindergärten und Seminare war wesentlich höher als die Durchführungsmöglichkeit. Inzwischen erfolgt eine weitere Unterstützung durch andere
Kinder und Jugendliche, dass die betroff Kinder und Jugendliche häufig in ihren Schulen erhebliche Nachteile bedingt durch das Asthma erleiden:

- Probleme der Konzentration und des Lernfortschrittes.

- Probleme der allergenbelasteten Klassenräume (Tierhaltung, Teppiche, Chemieunterricht), die ein erhöhtes Symptomrisiko für die Patienten ermöglichen. 
Tab. 9 Eigene Erfahrungen mit Asthma bronchiale; $\mathrm{n}=587$ (Angaben in \%)

\begin{tabular}{|c|c|c|c|}
\hline & ja & nein & keine Antwort \\
\hline Gibt es in Ihrer Klasse Kinder mit bekanntem Asthma bronchiale? & 37,4 & 57,5 & 5,1 \\
\hline Trat jemals im Unterricht bei einem Kind ein Asthmaanfall auf? & 15,2 & 83,6 & 0,2 \\
\hline Trat jemals im Rahmen des Sportunterrichtes des Kindes ein Asthmaanfall auf? & 14,5 & 72,0 & 13,5 \\
\hline Sind Sie selbst Elternteil eines asthmakranken Kindes? & 7,5 & 92,0 & 0,5 \\
\hline Leiden Sie selbst an Asthma bronchiale? & 7,5 & 91,6 & 0,9 \\
\hline
\end{tabular}

Tab. 10 Inwiefern glauben Sie die Informationen und Anregungen aus der Fortbildung in Ihrem Schulalltag umsetzen zu können? n= 587 (Angaben in \%)

\begin{tabular}{llllll}
\hline Untersuchung im Anschluss an das Seminar & $\begin{array}{l}\text { voll } \\
\text { anwendbar }\end{array}$ & $\begin{array}{l}\text { weitgehend } \\
\text { anwendbar }\end{array}$ & $\begin{array}{l}\text { weniger } \\
\text { anwendbar }\end{array}$ & $\begin{array}{l}\text { gar nicht } \\
\text { anwendbar }\end{array}$ & $\begin{array}{l}\text { keine } \\
\text { Antwort }\end{array}$ \\
\hline Lungendetektiv & 31,5 & 47,5 & 15,4 & 3,5 & 2,1 \\
Auslöser/-vermeidung & 21,5 & 57,0 & 19,4 & 0,5 & 1,6 \\
Notfallmanagement & 50,4 & 44,8 & 2,6 & 0,3 & 1,9 \\
\hline
\end{tabular}

Tab. 11 "Stellen Sie sich eine Notfallsituation in Ihrer Schule vor. Welche der folgenden (Organisations-)Schritte trauen Sie sich zu?“ $\mathrm{n}=587$ (Angaben in \%)

\begin{tabular}{|c|c|c|c|c|c|}
\hline Untersuchung im Anschluss an das Seminar & völlig & weitgehend & weniger & gar nicht & keine Antwort \\
\hline persönlich Ruhe bewahren & 29,8 & 65,4 & 4,4 & 0,2 & 0,2 \\
\hline Lungendetektiv/Peak Flow & 31,3 & 47,7 & 15,6 & 2,6 & 2,8 \\
\hline Lippenbremse/Kutschersitz & 80,9 & 18,4 & 0,3 & 0,2 & 0,2 \\
\hline Getränk anbieten & 78,8 & 15,8 & 3,8 & 1,1 & 0,5 \\
\hline Inhalation bronchienerweiternder Medikamente & 29,6 & 52,1 & 14,7 & 2,4 & 1,2 \\
\hline orale Medikamentengabe & 31,6 & 44,1 & 18,7 & 4,0 & 1,6 \\
\hline Klassenkameraden beruhigen & 38,6 & 55,6 & 4,7 & 0,2 & 0,9 \\
\hline Arzt verständigen & 86,4 & 12,4 & 0,7 & 0,2 & 0,3 \\
\hline
\end{tabular}

Tab.12 Maßnahmen zur Betreuung asthmakranker Kinder müssen in den Schulalltag integrierbar sein. Wie sieht es mit der Umsetzung der folgenden Punkte an Ihrer Schule aus? n= 587 (Angaben in \%)

\begin{tabular}{lllllc}
\hline Untersuchung im Anschluss an das Seminar & gut umsetzbar & umsetzbar & schlecht umsetzbar & nicht umsetzbar & keine Antwort \\
\hline Aushändigen von Notfallplänen & 73,4 & 23,1 & 1,6 & 0 & 1,9 \\
Gesamtkonferenz Aspekt Gesundheit & 5,6 & 52,6 & 33,4 & 4,5 & 3,9 \\
Fachgerecht eingerichtete Ruheräume & 5,4 & 34,6 & 45,5 & 11,0 & 9,1 \\
in gesundheitlichen Fragen ausgebildete Lehrer & 5,2 & 44,2 & 37,1 & 1,6 & 3,5 \\
Notfallpläne im Schulsekretariat & 39,2 & 47,2 & 8,2 & 2,3 & 2,6 \\
Notfallkoffer an zentraler Stelle & 36,9 & 49,1 & 9,1 & 2,1 & 2,6 \\
Erreichbarkeit der Eltern & 28,9 & 52,6 & 13,8 & 31,8 & 61,5 \\
Sanierung des Schulgebäudes & 1,2 & 16,1 & 44,4 &
\end{tabular}

Sponsoren, so dass auch im Schuljahr 2000/2001 weitere Schulen ein derartiges Seminarangebot erhalten konnten. Auch diese Seminare werden fortlaufend weiter ausgewertet.

Die vorliegenden Daten beziehen sich auf die 40 teilnehmenden Einrichtungen ( $n=648$ Teilnehmer). Die Ergebnisse zeigen, dass ein Seminar, abgehalten in der Schule, möglichst das ganze Kollegium mit einem Schlüssel von einem Trainer auf 15 bis 20 Lehrer durchführbar ist und auch dazu führt, dass durch eine praktisch orientierte Fortbildung das gesamte Kollegium in den gleichen Weiterbildungsstand bezüglich der Betreuung asthmabetroffener Kinder gebracht werden kann. Diese Vorgehen scheint somit effektiver als überregionale Veranstaltungen mit Teilnahme nur einzelner Lehrer. Die 
Seminarinhalte sind in den Schulalltag aus Sicht der Teilnehmer integrierbar. Das Zutrauen der Lehrer für die Umsetzung der erlernten Inhalte, insbesondere den Einsatz der Maßnahmen eines Notfallplanes ist befriedigend bis gut. Der Langzeiteffekt des Seminars soll über eine Nachbefragung der teilnehmenden Lehrer 12 Monate nach Durchführung des Seminars überprüft werden [Einsatz des Fragebogens nach [17]].

Insgesamt ist es sinnvoll, die Weiterbildung von Lehrern und Erziehern bezüglich des Umganges mit einer chronischen Erkrankung durchzuführen. Es wurde für die vorliegende Studie bewusst Asthma bronchiale gewählt, da jeder Lehrer je Unterrichtsstunde bei einer Klassenfrequenz von 30 Schülern ca. 2 bis 5 Schüler mit Asthma bronchiale im Klassenraum hat. Somit war primär ein Interesse und auch eine hohe Relevanz für den Schulalltag zu erwarten.

Sollte die Einjahreskatamnese zeigen, dass auch ein langfristiger Effekt des Seminars erreicht werden konnte, so scheint es sinnvoll zu klären, ob zumindestens für häufige chronische Erkrankungen im Kindes- und Jugendalter ein entsprechendes Weiterbildungsangebot im Rahmen der Referendariatsausbildung erfolgt. Das von uns durchgeführte Projekt wurde gesponsert durch eine pharmakologische Firma. Die Kosten für ein Seminar von 3 Zeitstunden sind durchaus vom Umfang her kalkulierbar und somit auch in die Referendariatsbildung integrierbar. Eine Durchführung eines derartigen Seminars nach Abschluss der Lehrerausbildung ist von der Finanzierbarkeit her sicherlich sehr viel schwieriger (fehlender Etat im Budget der Bezirksregierungen). Daneben ist eine Weiterbildung für möglichst alle angehenden Lehrer im Rahmen ihrer eigentlichen Ausbildung der möglicherweise sinnvollere Ansatz.

Der Einbezug von ganzen Kollegien/Seminaren im Rahmen der Fortbildung ist dabei eine sinnvolle Alternative zu Fortbildungsvorträgen für einzelne interessierte Lehrer, die unter Umständen mit großen Schwierigkeiten die Fortbildungsinhalte im Schulalltag umsetzen können. Bundesweit gibt es inzwischen genügend ausgebildete Asthmatrainer die für ein derartiges Weiterbildungsangebot an Lehrer/Erzieher/Schulen prinzipiell zur Verfügung stehen.

\section{Danksagung}

Der Firma Glaxo Wellcome wird für die finanzielle Unterstützung dieser Studie gedankt.

\section{Literatur}

${ }^{1}$ v. Mutius E. Münchener Asthma- und Allergiestudie. Münchener Medizinische Wochenschrift 1991; 133: 675-680

${ }^{2}$ Wijst N. Epidemiologie von Asthma im Kindesalter im internationalen Vergleich. Allergologie 1996; 19: 234-240

${ }^{3}$ Berdel D, Reinhardt D, Hofmann D, Leupold W, Lindemann H. Therapieempfehlung der Gesellschaft Pädiatrische Pneumologie zur Behandlung des Asthma bronchiale bei Kindern und Jugendlichen. Monatsschrift Kinderheilkunde 1998; 146: 492 - 497

${ }^{4}$ Gebert N, Hümmelink R, Könning J, Staab D, Schmidt S, Szczepanski R, Runde B, Wahn U. Efficacy of a self-management program for childhood asthma - A prospective controlled study. Patient Education and Counseling 1998; 35: 213-220
${ }^{5}$ Szczepanski R, Lecheler J. Standard- und Qualitätssicherung der Asthmaschulung im Kindes- und Jugendalter. Prävention und Rehabilitation 1995; 7: 1-41

${ }^{6}$ Szczepanski R, Gebert N, Hümmelink R, Könning J, Schmidt S, Runde B, Wahn U. Ergebnis einer strukturierten Asthmaschulung im Kindes- und Jugendalter. Pneumologie 1996; 50: 544-548

${ }^{7}$ Arbeitsgemeinschaft Asthmaschulung im Kindes- und Jugendalter e.V. Qualitätssicherung in der Asthmaschulung von Kindern und Jugendlichen. München: Zuckschwerdt, 2001

${ }^{8}$ Szczepanski R, Petermann F, Freidel K, Becker PN, Gebert N, LobCorzilius T. Die Wirksamkeit der Asthmaschulung bei Kindern und Jugendlichen. kinderarzt 1998; 29: 1201 -1208

${ }^{9}$ Lob-Corzilius T, Petermann F. Asthmaschulung - Wirksamkeit bei Kindern und Jugendlichen. Weinheim: Beltz, 1997

${ }^{10}$ Scholtz W, Haubrock M, Lob-Corzilius T, Gebert N, Wahn U, Szczepanski R. Kostennutzenuntersuchung bei ambulanten Schulungsmaßnahmen für asthmakranke Kinder und ihre Familien. Pneumologie 1996; 50: 537-543

${ }^{11}$ Gibson PG, Henry RL, Vimpany GV, Halliday J. Asthma knowledge, attitudes and quality of life in adolescents. Archives of Disease in Childhood 1995; 73: 321 - 326

12 Hofmann E et al. Über die Häufigkeit des Anstrengungsasthmas im Kindesalter. Monatsschrift Kinderheilkunde 1982; 107: 130

${ }^{13}$ Lecheler J, Gauer S. Schuldefizite chronisch asthmakranker Kinder und Jugendlicher. Monatsschrift Kinderheilkunde 1991; 139: $69-72$

${ }^{14}$ Bevis M, Taylor B. What do school teachers know about asthma? Archives of Disease in Childhood 1990; 65: 622-625

${ }^{15}$ Fillmore EJ, Jones N, Blankson JM. Achieving treatment goals for schoolchildren with asthma. Archives of Disease in Childhood 1999; 77: 420-422

${ }^{16}$ French DJ, Carroll A. Western Australian primar school teacher\&s knowledge about childhood asthma and its management. Journal of Asthma 1997; 34: 469-475

17 Stohlhofer B, Lahrmann H, Frank W, Zwick H. Erhebung des Wissensstandes von Wieder Volksschullehrerinnen über Asthma bronchiale bei Kindern. Pneumologie 1998; 52: 406-411

${ }^{18}$ Friede G. Beschreibung und Analyse eines Projektes aus der Asthmaschulung am Beispiel von Lehrerfortbildungen. Osnabrück: Unveröffentlichte Hausarbeit, 1997

${ }^{19}$ Friede G. Auswertung des Projektes „Lehrerfortbildung zum Thema Asthma bronchiale an allgemeinbildenden Schulen“. Osnabrück: Unveröffentlichte Hausarbeit, 1998

${ }^{20}$ Theiling S, Szczepanski R, Lob-Corzilius T. Der „Luftiku(r)s“. 3. Auflage. Stuttgart: Georg Thieme Verlag, 2001

${ }^{21}$ Szczepanski R. Schulungsprogramme und andere complianceunterstützende Maßnahmen. In: Rieger C, v. d. Hardt H, Sennhauser F, Wahn U, Zach M (Hrsg.). Pneumologie des Kindes- und Jugendalters. Wien, Heidelberg: Springer, 1999: 768-781

Dr. med. R. Szczepanski

Kinderhospital Osnabrück

Iburger Str. 187

49082 Osnabrück 